\title{
Artículos de datos
}

\section{Hormigas asociadas a fragmentos de bosque seco y ambientes urbanos de Santa Marta, Colombia}

\author{
Ants associated with dry forest fragments and urban \\ environments in Santa Marta, Colombia
}

Lina María Ramos Ortega $\oplus^{\square} \square$, Hubert Sierra ${ }^{\circledR} \square$, Johan D. Roncallo ${ }^{\square} \square$, Roberto J. Guerrero ${ }^{\circledR} \square$

\section{Resumen}

Se presenta una base de datos de acceso público a través del Sistema de Información de Biodiversidad en Colombia (SIB), de las hormigas asociadas a fragmentos de bosque seco y ambientes urbanos de Santa Marta, Colombia. Los métodos de captura incluyeron la instalación de trampas de caída, cebos de proteína y carbohidratos, extracción por el método Winkler de las hormigas asociadas a la hojarasca y la recolección manual de aquellas que forrajeaban sobre la vegetación arbórea. El conjunto de datos contiene 54323 registros, distribuidos en siete subfamilias, 42 géneros, 88 especies y nueve morfoespecies. El presente listado es una herramienta para incentivar el estudio y conservación de las hormigas en áreas urbanas.

Palabras clave. Conservación de la biodiversidad. Ecología urbana. Especies exóticas. Formicidae. Patrones ecológicos.

\section{Abstract}

A public access database of ants associated with dry forest fragments and urban environments in Santa Marta, Colombia is made available through the Sistema de Información de Biodiversidad en Colombia (SIB) is presented. The collection methods included mainly pit-fall traps, baits, extraction of the ants from the litter by the Winkler method, and the manual collection of those that foraged on arboreal vegetation. The data set contains 54323 records, distributed in seven subfamilies, 42 genera, 88 species, and nine morphospecies. This list is a tool to promote the study and conservation of ants in urban areas.

Keywords. Biodiversity conservation. Ecological patterns. Exotic species. Formicidae. Urban ecology. 


\section{Introducción}

Debido a la expansión acelerada de las zonas urbanas, uno de los principales desafíos ambientales es comprender cómo este proceso afecta a la biodiversidad (Turrini \& Knop, 2015). Aunque la urbanización es una de las principales causas de pérdida de especies nativas, la alta heterogeneidad espacial en el hábitat urbano, producida por diferentes usos de la tierra, puede resultar en algunos casos en incrementos de la diversidad a nivel local (McKinney, 2002). Las ciudades, con su alto grado de transformación del paisaje pueden albergar una cantidad considerable de las especies de una región, así como promover escenarios de conservación y espacios para la educación ambiental (MacGregor-Fors \& Zuria, 2019).

Las hormigas constituyen uno de los grupos de insectos más conspicuos en los ecosistemas terrestres y se encuentran comúnmente asociados a los entornos urbanos donde habitan de forma exitosa, encontrándose en una amplia variedad de sitios, que abarcan desde zonas verdes, hasta espacios domiciliarios (p. ej., cocinas) y hospitales (Chacón de Ulloa et al., 2019). Entre las razones que explican el éxito de las hormigas en las ciudades están la gran variedad de recursos alimenticios que utilizan (Blüthgen \& Feldhaar, 2010), la capacidad de anidar en suelos compactos y superficies con cobertura gris (Dáttilo \& MacGregor-Fors, 2021), y una alta tolerancia al estrés por calor (Angilletta et al., 2007).

En general, las ciudades son espacios altamente heterogéneos, en donde pueden generarse gradientes entre los sitios urbanizados y el conjunto de áreas verdes (p. ej., fragmentos de bosque, parques, lotes baldíos, entre otros). De esta forma, el efecto de la urbanización sobre la comunidad de hormigas parece ser el resultado del contexto espacial y de la capacidad de adaptación de las especies nativas a las nuevas condiciones de hábitat que impone el ambiente urbano. La ciudad de Santa Marta no es ajena a esta heterogeneidad, encontrándose inmersa en un contexto de bosque seco tropical, el cual es considerado como uno de los ecosistemas más amenazados y con un alto grado de degradación en Colombia, cuya distribución en la mayoría de los casos se da en forma de fragmentos o parches (García \& González, 2019). Por su parte, los estudios de la mirmecofauna del bosque seco en Colombia han estado enfocados en mayor medida hacia las zonas naturales (Fontalvo \& Solís, 2009; Chacón et al., 2012; Achury et al., 2020), dejando vacíos de información en las zonas urbanas.
La investigación que dio como resultado el conjunto de datos aquí presentados tuvo por objeto conocer la dinámica espacial y temporal de la diversidad taxonómica de hormigas en el ambiente urbano de Santa Marta, así como el impacto de la transformación del paisaje natural sobre la diversidad de este grupo de insectos (Ramos, 2021). Este estudio cubre una escala espacial y temporal amplia a nivel urbano. Además, se maximiza el uso de diferentes métodos de captura de hormigas para obtener una estimación fiable del inventario de este grupo. Por lo tanto, se pone a disposición información potencialmente relevante para la identificación de patrones ecológicos asociados a áreas urbanas de la ciudad de Santa Marta, así como promover la conservación de las hormigas en estos ambientes.

\section{Datos del proyecto}

Título. Diversidad de hormigas en ambientes urbanos de la ciudad de Santa Marta (Colombia)

\section{Nombre. Lina María Ramos Ortega}

Fuentes de financiación. El proyecto fue financiado con recursos de capacidad instalada a través de la Vicerrectoría de Investigación de la Universidad del Magdalena.

Descripción del área estudio. La recolección de la información se desarrolló en tres tipos de ambientes dentro de la zona urbana y periurbana de la ciudad de Santa Marta: parques distritales, parques temáticos y zonas naturales (fragmentos de bosque seco). Se realizaron cuatro muestreos entre los meses de octubre-diciembre de 2019 y enero de 2020, tratando de cubrir meses de alta y baja precipitación.

Descripción del proyecto. El proyecto estuvo orientado a conocer la dinámica espacial y temporal de la diversidad de hormigas en el ambiente urbano de la ciudad de Santa Marta, así como el impacto de la transformación del paisaje sobre la diversidad de este grupo de insectos. Otro aspecto importante en esta evaluación fue establecer si las zonas verdes de la ciudad (parques distritales y temáticos), son espacios que propician el mantenimiento y la conservación de la diversidad local.

\section{Cobertura taxonómica}

Descripción. El conjunto de datos contiene 54323 registros de la familia Formicidae (Insecta: Hymenoptera) correspondientes a 52946 especímenes, distribuidos en 
siete subfamilias, 42 géneros, 88 especies y nueve morfoespecies, provenientes de los muestreos realizados durante el desarrollo del proyecto "Diversidad de hormigas en ambientes urbanos de la ciudad de Santa Marta".

\section{Categorías}

Subfamilia. Dolichoderinae, Dorylinae, Ectatomminae, Formicinae, Myrmicinae, Ponerinae, Pseudomyrmecinae.

Género. Acromyrmex Anochetus, Azteca, Brachymyrmex Camponotus, Cardiocondyla, Cephalotes, Crematogaster, Cyphomyrmex, Dolichoderus, Dorymyrmex, Ectatomma, Forelius, Hypoponera, Kalathomyrmex, Labidus, Leptogenys, Megalomyrmex, Monomorium, Mycetomoellerius, Myrmicocrypta, Neivamyrmex, Nesomyrmex, Nylanderia, Odontomachus, Pachycondyla, Paratrachymyrmex, Paratrechina, Pheidole, Platythyrea, Pogonomyrmex, Pseudomyrmex, Rogeria, Sericomyrmex, Solenopsis, Strumigenys, Tapinoma, Temnothorax, Tetramorium, Thaumatomyrmex, Trichomyrmex, Wasmannia.

Especie. Acromyrmex octospinosus, Acromyrmex santschii, Anochetus inermis, Brachymyrmex cordemoyi, Brachymyrmex minutus, Camponotus blandus pronotalis, Camponotus zonatus, Camponotus coruscus, Camponotus lindigi, Cardiocondyla emery, Cephalotes femoralis, Cephalotes minutus, Cephalotes pellans, Cephalotes pusillus, Crematogaster abstinens, Crematogaster crinosa, Crematogaster distans, Crematogaster limata, Crematogaster obscurata, Crematogaster rochai, Crematogaster torosa, Cyphomyrmex flavidus, Cyphomyrmex rimosus, Dolichoderus diversus, Dorymyrmex biconis, Dorymyrmex tuberosus, Ectatomma ruidum, Ectatomma tuberculatum, Forelius damiani, Hypoponera clavatula, Hypoponera opacior, Kalathomyrmex emery, Labidus coecus, Leptogenys pubiceps, Leptogenys ritae, Megalomyrmex silvestrii, Monomorium pharaonis, Mycetomoellerius urichii, Mycetomoellerius zeteki, Myrmicocrypta buenzlii, Neivamyrmex iridescens, Nylanderia nodifera, Odontomachus bauri, Odontomachus ruginodis, Pachycondyla harpax, Paratrachymyrmex cornetzi, Paratrachymyrmex irmgardae, Paratrechina longicornis, Pheidole distorta, Pheidole fallax, Pheidole guajirana, Pheidole impressa, Pheidole indica, Pheidole inversa, Pheidole leptina, Pheidole praeusta, Pheidole radowszkowskii, Pheidole subarmata, Pheidole urbana, Platythyrea pilosula, Pogonomyrmex mayri, Pseudomyrmex boopis, Pseudomyrmex curacaensis, Pseudomyrmex eduardi, Pseudomyrmex elongatus, Pseudomyrmex gracilis, Pseudomyrmex simplex, Pseudomyrmex urbanus, Pseudomyrmex venustus, Rogeria curvipubens, Rogeria foreli, Sericomyrmex bondari, Solenopsis altinodis, Solenopsis bicolor, Solenopsis geminata, Solenopsis picea, Solenopsis whitfordi, Strumigenys dyseides, Strumigenys eggersi, Strumigenys elongata, Strumigenys spatula, Strumigenys tanimastax, Tapinoma melanocephalum, Temnothorax subditivus, Tetramorium lanuginosum, Tetramorium simillimum, Trichomyrmex destructor, Wasmannia auropunctata.

\section{Cobertura geográfica}

Descripción. Los registros provienen del muestreo de ocho parques distritales (P. Simón Bolívar, P. Avenida del Río, P. Gaira, P. de la Vida, P. Manzanares, P. Equidad, P. Los Almendros y P. Los Trupillos), cuatro parques temáticos (Campus de la Universidad del Magdalena, Jardín Botánico Quinta de San Pedro Alejandrino, Centro recreacional Teyuna y Parque del Agua) y cuatro zonas naturales (Parcela Bosque Seco Universidad del Magdalena, Fragmento de Bosque Seco Quinta de San Pedro Alejandrino, Reserva La Iguana Verde y Quebrada Seca).

Coordenadas. $11^{\circ} 10^{\prime} 11.17^{\prime \prime} \mathrm{N}$ y $11^{\circ} 14^{\prime} 45.17^{\prime \prime} \mathrm{N}$ latitud; $74^{\circ} 8^{\prime} 32.1^{\prime \prime} \mathrm{W}$ y $74^{\circ} 13^{\prime} 22.12^{\prime \prime} \mathrm{W}$ longitud. Los registros comprenden un intervalo altitudinal entre 1 y 100 m s.n.m.

\section{Cobertura temporal}

09 de octubre de 2019 - 23 de enero de 2020

\section{Datos de la colección}

Nombre de la colección. Centro de Colecciones Biológicas de la Universidad del Magdalena

Identificador de la colección. Registro Nacional de Colecciones: 207

\section{Identificador de la colección parental. CBUMAG:ENT}

Método de preservación de los especímenes. Ejemplar completo en Etanol

\section{Materiales y métodos}

Área de estudio La ciudad de Santa Marta, al norte de Colombia, presenta una extensión total de $2393.35 \mathrm{~km}^{2}$, de las cuales $55.10 \mathrm{~km}^{2}$ corresponden al área urbana 
(Geografía Urbana, 2020). La temperatura promedio anual es de $27^{\circ} \mathrm{C}$ y la precipitación promedio anual es de $608.8 \mathrm{~mm}$, con un régimen de lluvias unimodal (Rangel \& Carvajal-Cogollo, 2012). La cobertura vegetal predominante en estas zonas está caracterizada por bosques y matorrales de piso isomegatérmico (temperaturas altas y constantes durante todo el año), con caracteres xeromórficos pronunciados debido a que la temporada sin lluvias es más prolongada, alcanzando nueve meses al año (Carbonó et al., 2013; Hernández-Camacho \& Sánchez, 1992). De acuerdo con las características fisonómicas y florísticas, estas formaciones vegetales son definidas como las de un zonobioma subxerofítico tropical (Hernández-Camacho \& Sánchez, 1992). Los sitios de recolección de los datos y algunas de sus principales características se presentan en la Tabla 1.

Descripción del muestreo. Se realizaron cuatro muestreos entre los meses de octubre-noviembre de 2019 (época lluviosa) y diciembre de 2019-enero de 2020 (época seca). La elección de los métodos de captura y el número unidades de muestreo se hizo con base en las recomendaciones establecidas en Guerrero et al. (2019). En los parques de la ciudad, la recolección de las hormigas se hizo a través de dos tipos de cebos: atún (fuente de proteína; $\mathrm{BaP}$ ) y una mezcla de galleta y leche condensada (fuente de carbohidrato; BaC). Adicionalmente, se realizó la recolección manual de las hormigas que forrajean o habitan en la vegetación arbórea principalmente $(\mathrm{C})$. En las zonas naturales, además de los métodos descritos, se instalaron trampas pitfall (Pf) y se recolectó hojarasca para la separación de las hormigas usando sacos Winkler $(\mathrm{mW})$.

\section{Control de calidad}

Debido a la variedad de ambientes urbanos monitoreados, así como los métodos empleados para la captura de las hormigas, se establecieron códigos y etiquetas de recolección para hacer una clara diferenciación del ambiente (PD, PT, ZN) y método (BaC, $\mathrm{BaP}, \mathrm{Pf}, \mathrm{mW}, \mathrm{C})$; de esta forma, se minimizó la probabilidad de confusión de las muestras en campo y durante el proceso de separación, montaje e identificación en laboratorio. La identificación taxonómica se hizo hasta el nivel más bajo posible, para lo cual se emplearon claves taxonómicas especializadas. De igual forma, se hicieron comparaciones con colecciones de referencia depositadas en el CBUMAG. La información geográfica de cada registro se asoció a cada trampa o punto de muestreo donde fueron recolectados los especímenes, los cuales fueron georreferenciados con un GPSmap Garmin ${ }^{\circledR} 62$. Cada muestra se encuentra depositada en CBUMAG con su número de catálogo respectivo.

\section{Descripción de la metodología paso a paso}

1. Cada sitio fue georeferenciado antes del inicio de las actividades de campo. En los ambientes naturales se hizo la delimitación de los tres transectos de 100 m con la ayuda de una cuerda plástica, la cual fue marcada cada $10 \mathrm{~m}$ y en cada una de estas marcas se ubicó una trampa de caída o pitfall (vasos plásticos de 7 onzas o $207 \mathrm{ml}$ ), llenándolas hasta $1 / 3$ de su volumen con alcohol al $96 \%$; en cada zona natural se instalaron 30 trampas, las cuales se dejaron actuar por 72 horas

2. La recolección de la hojarasca se hizo dentro del área delimitada por los transectos. Se establecieron cuadrantes de $1 \mathrm{~m}^{2}$ y el material vegetal allí contenido fue pasado por un cernidor para descartar las fracciones más gruesas. El material fino fue depositado en bolsas de tela y llevadas hasta el laboratorio para el proceso final de extracción en los sacos Winkler. El tiempo de acción de las trampas fue de 48 horas.

3. La instalación de los cebos se hizo en los tres ambientes urbanos considerados en el estudio. Los cebos de proteína (atún) y carbohidratos (galleta + leche condensada) fueron depositados en trozos de cartulina $(10 \times 10 \mathrm{~cm})$ y dejados sobre la superficie del suelo con un tiempo de acción de 30 minutos. En los parques distritales y temáticos los cebos fueron dispuestos en las zonas de jardineras. Mientras que, en las zonas naturales, los cebos fueron instalados a lo largo de los transectos delimitados, cubriéndolos de forma proporcional. En cada sitio se instalaron 15 cebos de proteína y 15 cebos de carbohidratos. Las hormigas presentes en los cebos fueron depositadas en bolsas Whirl-Pak o viales plásticos, cada uno de ellos rotulados considerando el número y tipo de cebo. Todas las muestras fueron preservadas en alcohol al $96 \%$ y transportadas al laboratorio para su limpieza y separación.

4. La recolección de las hormigas que forrajeaban principalmente sobre la vegetación arbórea se hizo de forma manual. En cada sitio se hizo la inspección visual de la vegetación por el lapso de una hora y todo el material recolectado fue depositado en viales plásticos previamente rotulados y llenados con alcohol al $96 \%$. 5. El material recolectado en campo fue sometido a un proceso de limpieza para la eliminación de material vegetal y sedimentos en el caso de las muestras 
Tabla 1. Descripción de los ambientes urbanos monitoreados para el estudio de hormigas asociadas a fragmentos de bosque seco y ambientes urbanos de Santa Marta, Colombia.

Table 1. Description of the urban environments monitored for studying ants associated with dry forest fragments and urban environments in Santa Marta, Colombia.

\begin{tabular}{|c|c|c|c|}
\hline Ambiente & Coordenadas & $\begin{array}{c}\text { Área } \\
\text { (ha) }\end{array}$ & Características \\
\hline P. Simón Bolívar & $\begin{array}{l}11^{\circ} 14^{\prime} 41.856^{\prime \prime} \mathrm{N} \\
74^{\circ} 12^{\prime} 48.959^{\prime \prime} \mathrm{W}\end{array}$ & 1.0 & \multirow{8}{*}{$\begin{array}{l}\text { Los parques distritales se caracterizan por ser zonas de acceso li- } \\
\text { bre y de superficie variable. Las especies vegetales allí presentes } \\
\text { son una combinación de especies nativas (Tabebuia rosea, Prosopis } \\
\text { juliflora, Ceiba pentandra, Enterolobium cyclocarpum, entre otras) e } \\
\text { introducidas (Mangifera indica, Azadirachta indica, Terminalia cata- } \\
\text { ppa, Tamarindus indica, entre otras); exhibiéndose por lo general una } \\
\text { baja riqueza vegetal (10 especies en promedio). De igual forma, } \\
\text { presentan amplias superficies de concreto a su alrededor (bancas, } \\
\text { jardineras, etc). }\end{array}$} \\
\hline $\begin{array}{l}\text { P. Avenida del } \\
\text { Río }\end{array}$ & $\begin{array}{l}11^{\circ} 13^{\prime} 52.68^{\prime \prime} \mathrm{N} \\
74^{\circ} 11^{\prime} 8.843^{\prime \prime} \mathrm{W}\end{array}$ & 0.1 & \\
\hline WP. de Gaira & $\begin{array}{l}11^{\circ} 11^{\prime} 23.28^{\prime \prime} \\
74^{\circ} 13^{\prime} 22.115^{\prime \prime} \mathrm{W}\end{array}$ & 0.4 & \\
\hline P. de la Vida & $\begin{array}{l}11^{\circ} 12^{\prime} 43.2^{\prime \prime} \quad \mathrm{N} \\
74^{\circ} 11^{\prime} 20.615^{\prime \prime} \mathrm{W}\end{array}$ & 0.8 & \\
\hline P. de Manzanares & $\begin{array}{l}11^{\circ} 13^{\prime} 55.884^{\prime \prime} \mathrm{N} \\
74^{\circ} 12^{\prime} 38.123^{\prime \prime} \mathrm{W}\end{array}$ & 0.9 & \\
\hline P. de la Equidad & $\begin{array}{l}11^{\circ} 13^{\prime} 27.408^{\prime \prime} \mathrm{N} \\
74^{\circ} 11^{\prime} 50.82^{\prime \prime}\end{array}$ & 0.9 & \\
\hline P. Los Almendros & $\begin{array}{l}11^{\circ} 14^{\prime} 45.168^{\prime \prime} \mathrm{N} \\
74^{\circ} 11^{\prime} 42.827^{\prime \prime} \mathrm{W}\end{array}$ & 0.1 & \\
\hline P. Los Trupillos & $\begin{array}{l}11^{\circ} 13^{\prime} 42.996^{\prime \prime} \mathrm{N} \\
74^{\circ} 10^{\prime} 15.959^{\prime \prime} \mathrm{W}\end{array}$ & 1.3 & \\
\hline $\begin{array}{l}\text { Campus } \\
\text { Unimagdalena }\end{array}$ & $\begin{array}{ll}11^{\circ} 13^{\prime} 35.4^{\prime \prime} & \mathrm{N} \\
74^{\circ} 11^{\prime} 10.824^{\prime \prime} \mathrm{W} & \end{array}$ & 11 & \multirow{4}{*}{$\begin{array}{l}\text { El acceso a los parques temáticos es más restringido, con una mayor } \\
\text { área que en los parques distritales. Las especies vegetales son tam- } \\
\text { bién una combinación de especies nativas (Prosopis juliflora, Ceiba } \\
\text { pentandra, Quadrella odoratissima, Enterolobium cyclocarpum, entre } \\
\text { otras) e introducidas (Mangifera indica, Azadirachta indica, Delonix } \\
\text { regia, Spathodea campanulata, entre otras), aunque con un mayor } \\
\text { número de especies (18 especies en promedio). Presentan menos } \\
\text { superficie de concreto a su alrededor, ya que se promueve la interac- } \\
\text { ción positiva con la naturaleza que los rodea. Estos parques tienen } \\
\text { fines recreativos, culturales, y de educación ambiental. }\end{array}$} \\
\hline $\begin{array}{l}\text { Jardín Botánico } \\
\text { Quinta de San Pe- } \\
\text { dro Alejandrino }\end{array}$ & $\begin{array}{l}11^{\circ} 13^{\prime} 41.628^{\prime \prime} \mathrm{N} \\
74^{\circ} 10^{\prime} 41.376^{\prime \prime} \mathrm{W}\end{array}$ & 13 & \\
\hline $\begin{array}{l}\text { Centro } \\
\text { Recreacional } \\
\text { Teyuna }\end{array}$ & $\begin{array}{l}11^{\circ} 10^{\prime} 57.648^{\prime \prime} \mathrm{N} \\
74^{\circ} 13^{\prime} 0.191^{\prime \prime} \mathrm{W}\end{array}$ & 11.5 & \\
\hline P. del Agua & $\begin{array}{l}11^{\circ} 13^{\prime} 23.592^{\prime \prime} \mathrm{N} \\
74^{\circ} 10^{\prime} 28.523^{\prime \prime} \mathrm{W}\end{array}$ & 3.3 & \\
\hline $\begin{array}{l}\text { Parcela } \\
\text { Bosque Seco } \\
\text { Unimagdalena }\end{array}$ & $\begin{array}{l}11^{\circ} 13^{\prime} 16.068^{\prime \prime} \mathrm{N} \\
74^{\circ} 11^{\prime} 10.967^{\prime \prime} \mathrm{W}\end{array}$ & 2.8 & \multirow{4}{*}{$\begin{array}{l}\text { Corresponden a fragmentos de bosque seco ubicados dentro del } \\
\text { área urbana y periurbana de la ciudad y en todos los casos, estas } \\
\text { zonas son sujetos de conservación, investigación y educación am- } \\
\text { biental. Las especies vegetales allí presentes son características del } \\
\text { bosque seco con una riqueza vegetal entre 20-50 especies (Albizia } \\
\text { niopoides, Cordia alba, Acacia polyphylla, Pereskia guamacho, entre otras) } \\
\text { (Castellanos et al., 2019). }\end{array}$} \\
\hline $\begin{array}{l}\text { Parcela Bos- } \\
\text { que Seco Quin- } \\
\text { ta de San Pedro } \\
\text { Alejandrino }\end{array}$ & $\begin{array}{l}11^{\circ} 13^{\prime} 42.06^{\prime \prime} \quad \mathrm{N} \\
74^{\circ} 10^{\prime} 31.259^{\prime \prime} \mathrm{W}\end{array}$ & 22.0 & \\
\hline $\begin{array}{l}\text { Reserva La Iguana } \\
\text { Verde }\end{array}$ & $\begin{array}{l}11^{\circ} 10^{\prime} 11.172^{\prime \prime} \quad \mathrm{N} \\
74^{\circ} 10^{\prime} 29.639^{\prime \prime} \mathrm{W}\end{array}$ & 19.0 & \\
\hline Quebrada Seca & $\begin{array}{l}11^{\circ} 13^{\prime} 9.624^{\prime \prime} \quad \mathrm{N} \\
74^{\circ} 8^{\prime} 32.1^{\prime \prime}\end{array}$ & 6.0 & \\
\hline
\end{tabular}


obtenidas por los métodos pitfall y sacos Winkler, y restos de atún y galleta para el caso de los cebos. Las muestras fueron transferidas a viales plásticos y preservadas en alcohol al $96 \%$, manteniendo en todos los casos los rótulos designados en campo.

6. Para facilitar la identificación taxonómica de las hormigas se realizaron montajes en seco de los especímenes, empleando un estereoscopio Nikon SMZ745. Las claves taxonómicas empleadas correspondieron a los trabajos de Fernández et al. (2019b), Hoenle et al. (2020) y Camargo-Vanegas \& Guerrero (2020) y el recurso electrónico AntWeb Version 8.56. Los ejemplares fueron depositados en las Colecciones Biológica de la Universidad del Magdalena - CBUMAG.

\section{Resultados}

\section{Descripción del conjunto de datos}

La mirmecofauna recolectada en los ambientes urbanos de Santa Marta representan alrededor del $64 \%$ de las subfamilias registradas para el Neotrópico y Colombia, así como el 40 \% de los géneros en Colombia (Fernández et al., 2019a). De los 97 taxones determinados, 88 fueron identificadas a nivel de especie, correspondiendo al $8 \%$ de los registros actuales para el país (Fernández et al., 2019a; García et al., 2020). Las subfamilias Myrmicinae, Ponerinae, Formicinae y Pseudomyrmecinae contribuyeron con cerca del $88 \%$ de la riqueza de hormigas, tendencia que se mantuvo relativamente constante entre los ambientes urbanos evaluados (Figura 1). Los géneros Pheidole, Cephalotes, Crematogaster, Pseudomyrmex, Camponotus, Solenopsis y Strumygenys aportaron alrededor del $50 \%$ de las especies registradas. Por su parte, Dorymyrmex, Ectatomma, Odontomachus, entre otros géneros, tuvieron entre 2 y 3 especies (Figura 2); mientras que un total de 12 géneros registraron una única especie. Las especies con mayores frecuencias de captura correspondieron a Ectatomma ruidum (44 \%), Dorymyrmex biconis (19\%), Pheidole indica (13 $\%)$, Pheidole fallax (12\%) y Trichomyrmex destructor (10 \%).

En este estudio, más del $90 \%$ de los taxones fueron identificadas a un nivel específico, permitiendo reconocer nuevos registros de hormigas para el país dentro de los géneros Hypoponera, Myrmicocrypta, Solenopsis y Strumigenys. De igual forma, se identificó la presencia de hormigas exóticas, algunas de amplia distribución en Colombia como es el caso de Paratrechina longicornis, Monomorium pharaonis y Tapinoma melanocephalum; mientras que las especies Pheidole indica, Trichomyrmex destructor, Tetramorium lanuginosum, Tetramorium simillimum

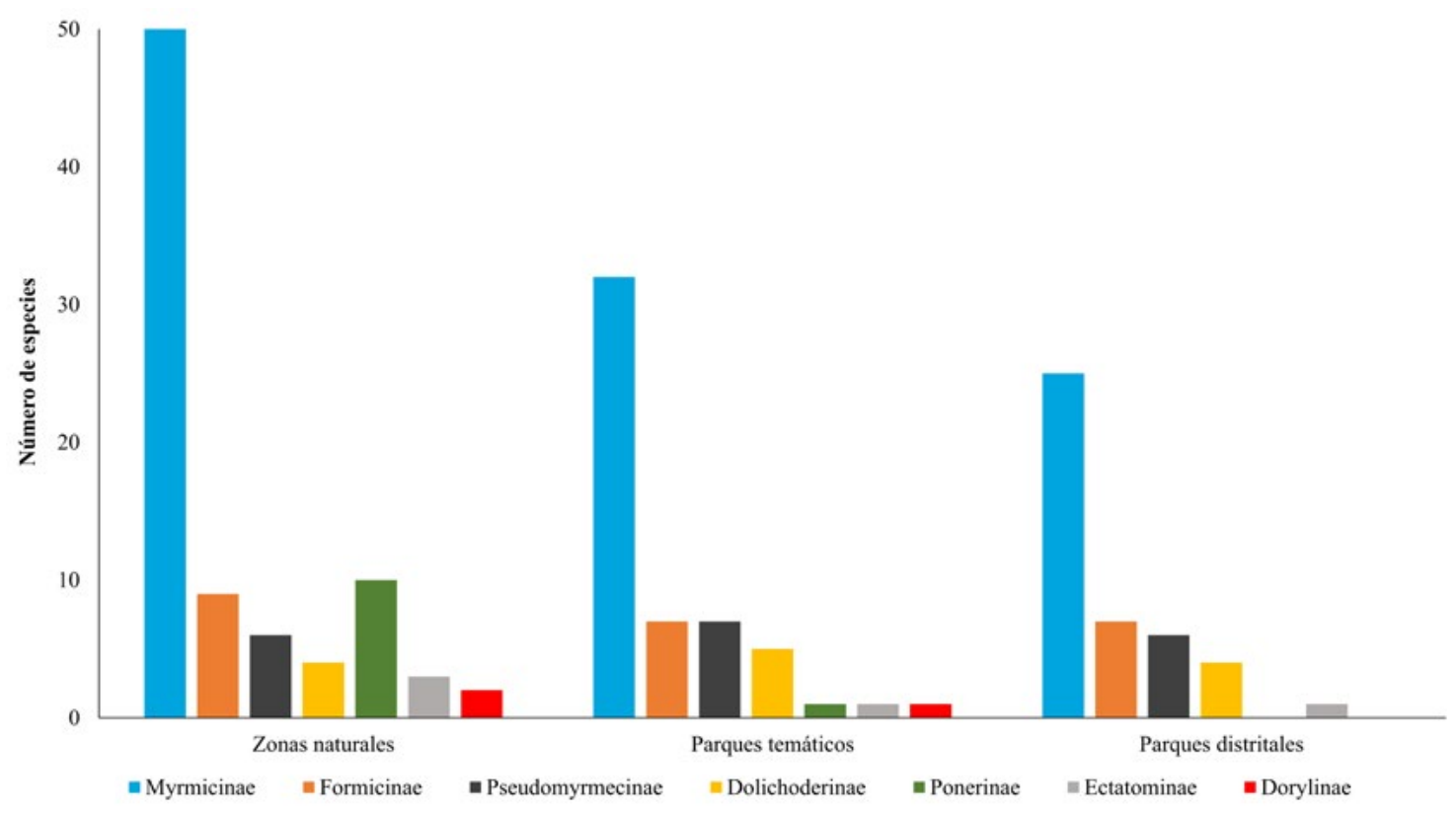

Figura 1. Número de especies de hormigas por subfamilia en fragmentos de bosque seco y ambientes urbanos de Santa Marta, Colombia.

Figure 1. Number of ant species by subfamily in dry forest fragments and urban environments in Santa Marta, Colombia. 


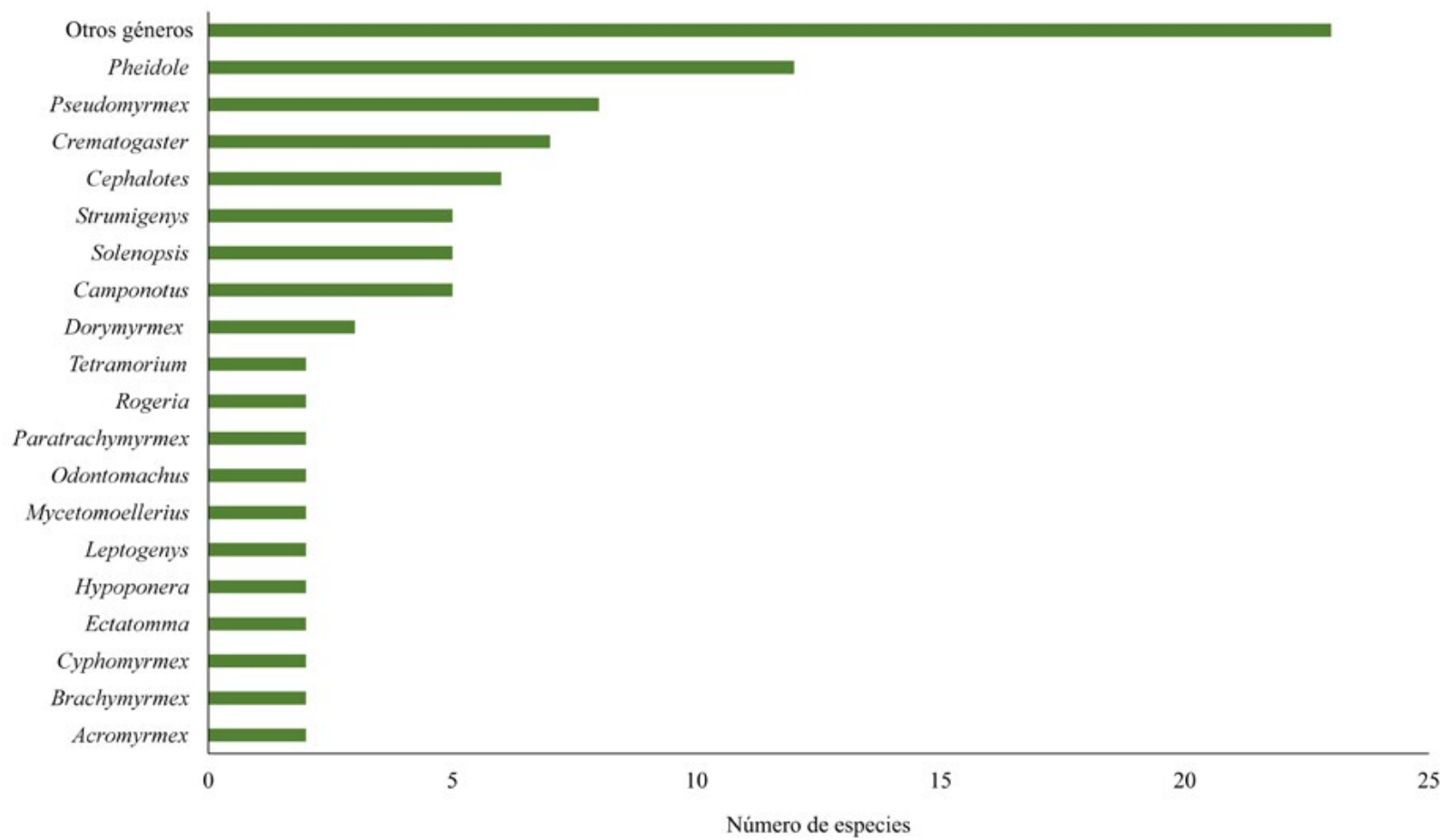

Figura 2. Número de especies de hormigas por género en fragmentos de bosque seco y ambientes urbanos de Santa Marta, Colombia. La categoría "Otros géneros" contiene a todos aquellos taxones genéricos con una sola especie.

Figure 2. Number of ant species by genus in dry forest fragments and urban environments in Santa Marta, Colombia. The category “Other genera" contains all those gener with a single species.

y Cardiocondyla emeryi han sido registradas en algunas pocas localidades en el país (Dekoninck et al., 2019).

Debido a la variedad de microhábitat en donde pueden establecerse las hormigas, la elección de los métodos de captura es un aspecto trascendental en los estudios de diversidad y de ecología en general. Para el conjunto de datos aquí presentado, los tres métodos de captura empleados brindaron tanto información única como complementaria, del inventario de las hormigas asociadas al ambiente urbano. Los métodos pitfall (Pf) y recolección manual (CM) arrojaron la mayor cantidad de especies, seguido del método para las hormigas de la hojarasca $(\mathrm{mW})$ (Tabla 2). Con relación a los cebos, el de proteína fue el que capturó una mayor riqueza. Por su parte, el número de especies que fueron recolectadas solo con alguno de los métodos corresponde al $24 \%$ de la riqueza total del estudio, lo que resalta la importancia de utilizar métodos de captura variados y que abarquen diferentes microhábitats en los ambientes evaluados. Las especies más frecuentes entre los métodos corresponden a Ectatomma ruidum, Crematogaster crinosa, Acromyrmex santschii, Solenopsis bicolor, Pheidole guajirana, entre otras (Tabla 2).
URL del recurso. Para acceder a la última versión del conjunto de datos:

IPT. https:/ / doi.org/10.15472/2cdkza

Portal de datos. http:// datos.biodiversidad.co/ dataset/b56fbfec-cf01-458a-b772-2df2c8403201

Portal GBIF. https: / / www.gbif.org/dataset/ b56fbfec-cf01-458a-b772-2df2c8403201

Nombre. Diversidad de hormigas en ambientes urbanos de la ciudad de Santa Marta

Idioma. Español

Codificación de caracteres. UTF-8

URL del archivo. Para acceder a la versión del conjunto de datos descrita en este artículo:

https:/ / ipt.biodiversidad.co/biota/

resource?r=umagdalena_hormigasurbanas

Formato del archivo. Darwin Core

Versión del formato del archivo. 1.4

Nivel de jerarquía. Dataset

Fecha de publicación de los datos. 2021-08-02

Idioma de los metadatos. Español

Fecha de creación de los metadatos. 2020-07-29

Licencia de uso. Extraída del recurso publicado a través del SiB Colombia. 
Tabla 2. Riqueza, número de especies exclusivas y frecuentes según el método de captura, de las hormigas que habitan en los ambientes urbanos de Santa Marta, Colombia.

Table 2. Richness, number of exclusive and frequent species, according to the collection method, of the ants that inhabit urban environments of Santa Marta, Colombia.

\begin{tabular}{|c|c|c|c|}
\hline Método de captura & Riqueza & $\begin{array}{l}\text { No. especies } \\
\text { exclusivas }\end{array}$ & Especies frecuentes \\
\hline $\begin{array}{l}\text { Cebo carbohidrato } \\
(\mathrm{BaC})\end{array}$ & 40 & 0 & \multirow{2}{*}{$\begin{array}{l}\text { Ectatomma ruidum, Dorymyrmex biconis, Pheidole indica, Trichomyr- } \\
\text { mex destructor }\end{array}$} \\
\hline Cebo proteína $(\mathrm{BaP})$ & 50 & 1 & \\
\hline Colecta manual (CM) & 63 & 7 & $\begin{array}{l}\text { Crematogaster crinosa, Brachymyrmex minutus, Camponotus lindi- } \\
\text { gi, Paratrechina longicornis }\end{array}$ \\
\hline $\begin{array}{l}\text { Trampas de caída } \\
\text { (Pf) }\end{array}$ & 67 & 6 & $\begin{array}{l}\text { Ectatomma ruidum, Acromyrmex santschii, Pheidole fallax, Campo- } \\
\text { notus zonatus, Pogonomyrmex mayri, Pheidole guajirana }\end{array}$ \\
\hline $\begin{array}{l}\text { Extracción Winkler } \\
(\mathrm{mW})\end{array}$ & 60 & 9 & $\begin{array}{l}\text { Solenopsis bicolor, Pheidole guajirana, Solenopsis picea, Anochetus } \\
\text { inermis, Hypoponera opacior }\end{array}$ \\
\hline
\end{tabular}

\section{Agradecimientos}

Gracias a las directivas de la Quinta San Pedro Alejandrino y de la parcela de BST de la Unimagdalena por permitir el acceso. A Luz Adriana Velazco de la Reserva Natural Iguana Verde y Libardo López en Quebrada Seca por el acceso al BST dentro de sus predios. Al Centro de Colecciones Científicas de la Unimagdalena por el apoyo logístico. Los autores expresan no tener ningún tipo de conflicto de intereses.

\section{Referencias}

Achury, R., Chacón de Ulloa, P., Arcila, A. \& Suárez, A. (2020). Habitat disturbance modifies dominance, coexistence, and competitive interactions in tropical ant communities. Ecological entomology.

https:/ / doi.org/110.1111/een.12908

Angilletta, M. J., Wilson, R. S., Niehaus, A. C., Sears, M. W., Navas, C. A., \& Ribeiro, P. L. (2007). Urban physiology: city ants possess high heat tolerance. PLoS ONE, 2(2), 4.

https:// doi.org/10.1371/journal.pone.0000258

Blüthgen, N., \& Feldhaar, H. (2010). Food and shelter: How reosurces influence ant ecology. En Oxford biology. Ant ecology. (Pp. 115-136). Oxford University Press.

Camargo-Vanegas, J. J., \& Guerrero, R. J. (2020). Las hormigas Pheidole (Formicidae: Myrmicinae) en el bosque seco tropical de Santa Marta, Colombia. Revista Colombiana de Entomología, 46(2), e8433.
Carbonó-Delahoz, E., Barros-Barraza, A. \& Jiménez-Vergara, J. (2013). Cactaceae de Santa Marta, Magdalena, Colombia. Revista de la Academia Colombiana de Ciencias Exactas, Físicas y Naturales, 37(143), 177-187.

Castellanos Barliza, J., Barranco Pérez, W. \& León Peláez, J. D. (2019). Evaluación ecológica de un fragmento urbano de bosque seco. Universidad del Magdalena. https:/ / doi.org/10.21676/9789587462128

Chacón de Ulloa, P., Montoya-Lerma, J., Abadía, J. C., Rodríguez, J. \& Castaño-Quintana, K. (2019). Hormigas urbanas. En Hormigas de Colombia. (Pp. 11711185). Universidad Nacional de Colombia.

Chacón de Ulloa, P., Osorio-García, A. M., Achury, R. \& Bermúdez-Rivas, C. (2012). Hormigas (Hymenoptera: Formicidae) del Bosque seco Tropical (Bs-T) de la cuenca alta del río Cauca, Colombia. Biota Colombiana, 13(2), 165-181.

Dáttilo, W. \& MacGregor-Fors, I. (2021). Ant social foraging strategies along a Neotropical gradient of urbanization. Scientific Reports, 11(6119), 9. https:/ / doi.org/10.1038/s41598-021-85538-2

Dekoninck, W., Wauters, N. \& Delsinne, T. (2019). Hormigas invasoras en Colombia. En Hormigas de Colombia. (Pp. 1149-1169). Universidad Nacional de Colombia.

Fernández, F., Guerrero, R. \& Delsinne, T. (2019a). Filogenia y sistemática de las hormigas neotropicales. En Hormigas de Colombia. (Pp. 57-89). Universidad Nacional de Colombia.

Fernández, F., Guerrero, R. \& Delsinne, T. (Eds.). (2019b). Hormigas de Colombia. Universidad Nacional de Colombia. 
Fontalvo-Rodríguez, L. \& Solís-Medina, C. (2009). Ensamblaje de hormigas (Hymenoptera: Formicidae) en fragmentos de bosque seco en el complejo carbonífero El Cerrejón (La Guajira, Colombia). Revista Intropica, 4, 5-15.

García, E., Tocora, M. C., Fiorentino, G., Escárraga, M. E., Fernández, F. \& Guerrero, R. J. (2020). New records of ants (Hymenoptera: Formicidae) for Colombia. Biota Neotropica, 20(4), e20201088. https:/ / doi.org/10.1590/1676-0611-bn-2020-1088

García, H., \& González, R. (Eds.). (2019). Bosque seco Colombia: Biodiversidad y gestión. Instituto de Investigación de Recursos Biológicos Alexander von Humboldt.

Geografía Urbana. (2020). Plan de ordenamiento territorial Santa Marta documento de formulación. Alcaldía de Santa Marta.

Guerrero, R., Delsinne, T. \& Dekoninck, W. (2019). Métodos de recolección y curadurías. En Hormigas de Colombia. (Pp. 319-369). Universidad Nacional de Colombia.

Hernández-Camacho, J. \& Sánchez, H. (1992). Biomas terrestres de Colombia. En La Diversidad biologica de Iberoamerica. (Pp. 153-173). CYTED-D, Programa Iberoamericano de Ciencia y Tecnología para el Desarollo, Instituto de Ecología, A.C., Secretaría de Desarrollo Social.
Hoenle, P., Lattke, J., Donoso, D., von Beeren, C., Heethoff, M., Schmelzle, S., ... Blüthgen, N. (2020). Odontomachus davidsoni sp. Nov. (Hymenoptera, Formicidae), a new conspicuous trap-jaw ant from Ecuador. ZooKeys, 948, 75-105.

https:/ / doi.org/10.3897/zookeys.948.48701

MacGregor-Fors, I. \& Zuria, I. (2019). Ciudades vivas: Biodiversidad en ambientes urbanos. En C. Moreno (Ed.), La Biodiversidad en un mundo cambiante: Fundamentos teóricos y metodológicos para su estudio. (Pp. 113127). Universidad Autónoma del Estado de Hidalgo.

McKinney, M. L. (2002). Urbanization, Biodiversity, and Conservation. BioScience, 52(10), 883-890. https:// doi.org/10.1641/0006-3568(2002)052[0883 :UBAC]2.0.CO;2

Rangel, J. O. \& Carvajal-Cogollo, J. E. (2012). Clima de la región Caribe colombiana. En Diversidad Biótica XII: La región Caribe de Colombia. (pp. 67-129). Universidad Nacional de Colombia.

Ramos, L. M. (2021). Diversidad de hormigas en ambientes urbanos de la ciudad de Santa Marta. (Informe técnico). Vicerrectoría de Investigación, Universidad del Magdalena.

Turrini, T. \& Knop, E. (2015). A landscape ecology approach identifies important drivers of urban biodiversity. Global Change Biology, 21(4), 1652-1667. https://doi.org/10.1111/gcb.12825 


\section{Hubert Sierra}

Universidad del Magdalena

Santa Marta, Magdalena, Colombia

hubertsierraac@unimagdalena.edu.co

https:/ / orcid.org/0000-0002-2304-4466

Johan D. Roncallo

Universidad del Magdalena

Santa Marta, Magdalena, Colombia

jhoanroncallodj@unimagdalena.edu.co

https:/ / orcid.org/0000-0002-2075-3128

\section{Roberto J. Guerrero}

Universidad del Magdalena

Santa Marta, Magdalena, Colombia

rguerrero@unimagdalena.edu.co

https:/ / orcid.org/0000-0003-3244-2754

\section{Hormigas asociadas a fragmentos de bosque seco y ambientes urbanos de Santa Marta, Colombia}

Citación: Ramos-Ortega, L. M., Sierra, H., Roncallo, J. D. y Guerrero, R. J. (2022). Hormigas asociadas a fragmentos de bosque seco y ambientes urbanos de Santa Marta, Colombia. Biota Colombiana, 23(1), e978.

\section{https:/ / doi.org/10.21068/2539200X.978}

Recibido: 26 de mayo 2021

Aceptado: 6 de agosto 2021 